\title{
The Higgs boson in the MSSM in light of the LHC
}

\author{
D. Albornoz Vásquez ${ }^{1}$, G. Bélanger ${ }^{2}$, R. M. Godbole ${ }^{3}$ and A. Pukhov ${ }^{4}$ \\ 1) CNRS, UMR7095, Institut d'Astrophysique de Paris, F-75014 Paris, France \\ 2) LAPTH, Univ. de Savoie, CNRS, B.P.110, F-74941 Annecy-le-Vieux Cedex, France \\ 3) Centre for High Energy Physics, Indian Institute of Science, Bangalore, 560 012, India \\ 4) Skobeltsyn Inst. of Nuclear Physics, Moscow State Univ., Moscow 119992, Russia
}

\begin{abstract}
We investigate the expectations for the light Higgs signal in the MSSM in different search channels at the LHC. After taking into account dark matter and flavor constraints in the MSSM with eleven free parameters, we show that the light Higgs signal in the $\gamma \gamma$ channel is expected to be at most at the level of the SM Higgs, while the $h \rightarrow b \bar{b}$ from W fusion and/or the $h \rightarrow \tau \bar{\tau}$ can be enhanced. For the main discovery mode, we show that a strong suppression of the signal occurs in two different cases: low $M_{A}$ or large invisible width. A more modest suppression is associated with the effect of light supersymmetric particles. Looking for such modification of the Higgs properties and searching for supersymmetric partners and pseudoscalar Higgs offer two complementary probes of supersymmetry.
\end{abstract}

\section{Introduction}

The search for the Higgs boson is the primary goal of the Large Hadron Collider (LHC). The standard model (SM) Higgs in the mass range $141 \mathrm{GeV}<M_{h}<475 \mathrm{GeV}$ has been excluded by combined CMS and ATLAS analyses with integrated luminosities from 1 to $2.3 \mathrm{fb}^{-1}[1]$. In this mass range the primary search mode is $H \rightarrow W W^{(*)} \rightarrow l \nu l \nu$. For lower Higgs masses the main search channel is $g g \rightarrow H \rightarrow \gamma \gamma$ where both the production and the decay are loop-induced processes. Current limits range from roughly 1-4 times the SM cross section [1,2].1. The channels $H \rightarrow W W^{(*)} \rightarrow l \nu l \nu$ as well as $H \rightarrow Z Z^{(*)} \rightarrow l l l l$ still play a role in setting the global exclusion limit especially for masses near $140 \mathrm{GeV}$. Other search channels include associated Higgs and vector boson production followed by $H \rightarrow b \bar{b}$ with a limit on the cross section relative to the SM one, $\sigma / \sigma_{S M}<6$ for a Higgs boson mass in the range from 110 to $130 \mathrm{GeV}$ as well as $H \rightarrow \tau \tau$ from vector boson and gluon fusion with a limit on $\sigma / \sigma_{S M} \approx 4-6$ for the Higgs in the range from 110 to $130 \mathrm{GeV}$.

These results however do not yet constrain the light Higgs in the Minimal Supersymmetric Standard Model (MSSM). Indeed in the MSSM the radiative corrections can only raise the lightest Higgs mass to about $135 \mathrm{GeV}$. As for the SM Higgs, searches for the light Higgs in the MSSM rely mainly on $g g \rightarrow h \rightarrow \gamma \gamma$ with some contribution from the $h \rightarrow W W$ channel in the upper region of the mass range. The combined ATLAS and CMS limits for the $h \gamma \gamma$ channel exclude $\sigma / \sigma_{S M}<3.1$ in the mass range $110-121 \mathrm{GeV}$ and $\sigma / \sigma_{S M}<1.8$ in the $121-131 \mathrm{GeV}$ range where $\sigma\left(\sigma_{S M}\right)$ is the production cross section times branching ratio in the MSSM (SM). For the higher mass range, the collaboration have indeed become sensitive to $\sigma / \sigma_{S M} \approx 1$ with the integrated luminosity $\mathcal{L}=5 f b^{-1}[3]$ as was projected in [2]. The channel $H / A / h \rightarrow \tau \tau$ where the Higgses are produced via gluon fusion, or from b-quarks is the preferred channel for the search for the heavy Higgs doublet. This channel has in fact been used to set powerful limits on the heavy Higgs of the MSSM which has enhanced couplings at large values of $\tan \beta$ 4. Although more modest, the light Higgs couplings can also be enhanced. In the mass range

\footnotetext{
${ }^{1}$ The ATLAS collaboration has very recently reported an excess in this channel that could be compatible with a Higgs with a mass of $126 \mathrm{GeV}$ [3] while the CMS collaboration was not able to rule out the region between $117 \mathrm{GeV}<M_{h}<127 \mathrm{GeV}$ because of an excess of events.
} 
relevant for the light Higgs, limits on $\sigma / \sigma_{S M}$ range from 6-10 [4]. Note however that $\sigma_{S M}$ in the denominator includes only WW and gg fusion production processes.

The predictions for the supersymmetric Higgses can differ significantly from the SM ones for both production and decay. The Higgs couplings to vector bosons or to fermions (in particular to b-quarks) differ from their SM value in the limit where the mass of the pseudoscalar Higgs, $M_{A}$ is low [5 7]. Furthermore the loop-induced couplings $h g g$ and $h \gamma \gamma$ can receive important supersymmetric contributions from the chargino and stop sector. Finally the mass of the lightest neutralino could be below $M_{h} / 2$ thus leading to invisible decays of the light Higgs and to reduced rates for all visible Higgs decays. Note that for kinematical reasons this occurs mainly in models with non-universal couplings where very light neutralinos are allowed (in the constrained MSSM, LEP limits imply a neutralino above $46 \mathrm{GeV}[8]$ ).

The implications of supersymmetry on Higgs searches have been extensively studied over the years. The effect of top squarks on loop-induced process on the Higgs signal showed that large corrections can be found especially for light stops [9-14]. The effect of charginos on the two photon width of the Higgs was shown to be more modest [15, 16]. Changes in the $h \rightarrow b \bar{b}$ partial width due to supersymmetric corrections can enhance or suppress the branching fraction into two-photons [5, 11]. The modification of the $h \rightarrow b \bar{b}$ partial width by introducing an extra singlet that mixes with the Higgs can also enhance the two-photon branching fraction in the NMSSM [17]. More generally, in the MSSM with new degrees of freedom (BMSSM), the modification of the Higgs couplings were shown to lead to potentially suppressed or enhanced signals in all channels $18-20]$.

Invisible Higgs decays were examined in the framework of the MSSM with non-universal gaugino masses [16, 21] as well as in the R-parity violating MSSM [22,23], in the MSSM with a mixed sneutrino 24 26] in the NMSSM 27,28] or in the NMSSM with sneutrino dark matter [29]. For the latter the Higgs is made invisible by decaying into modes not searched for in the standard channels, typically into other light Higgses.

In addition to the above, invisible Higgs decays exist in many other models with a light dark matter candidate, including those with a scalar dark matter [30 32], a hidden sector [33 or in higher dimensional models [34, 35]. More generally the Higgs can become invisible in models with extra singlets [36, 37] or with an extra neutrino [38. Dedicated searches for the invisible Higgs were advocated at the LHC in either vector boson fusion [39, 40] or in associated vector boson production [40,41], see also [42,44]. Methods for measuring the invisible width were suggested 45, 46].

In this paper we reexamine the case of the Higgs production and decays in the MSSM with eleven free parameters. We use a MCMC approach to constrain the parameter space and take into account constraints from B-physics, $(g-2)_{\mu}$, neutralino relic density, LEP limits on supersymmetric particles, on the $\mathrm{Z}$ invisible width and on associated neutralino LSP production as well as limits from Higgs searches at colliders. We further impose additional constraints from dark matter searches : direct detection limits from XENON100 [47] and the photon flux from dwarf Spheroidal galaxies (dSph) measured by FermiLAT [48]. We consider only scenarios where the LSP is a neutralino since a sneutrino LSP is severely constrained by direct detection limits [49]. We then examine the consequences for $g g \rightarrow h \rightarrow \gamma \gamma, h \rightarrow \tau \bar{\tau}$ and $h \rightarrow b \bar{b}$ decays as well as for invisible Higgs decays. We find that in general the $g g \rightarrow h \rightarrow \gamma \gamma$ is comparable or somewhat suppressed as compared to the SM Higgs of the same mass. However large suppressions can also occur, we show that the suppressions are associated with the presence of some light particles thus highlighting the complementarity between SUSY and light/heavy Higgs searches.

This paper is organized as follows. Section 2 presents briefly the model and the constraints used in the fit. The observables linked to the light Higgs are presented in section 3. Our resuts are summarised in section 4 for each main Higgs decay channel. Benchmarks are also proposed. 
Section 5 contains our conclusions.

\section{Model and constraints}

We consider the MSSM with eleven free parameters defined at the electroweak scale, as in [50]:

$$
M_{1}, M_{2}, M_{3}, \mu, \tan \beta, M_{A}, M_{\tilde{l}_{L}}, M_{\tilde{l}_{R}}, M_{\tilde{q}_{1,2}}, M_{\tilde{q}_{3}}, A_{t} .
$$

We assume minimal flavour violation, two common soft masses $M_{\tilde{l}_{L}}$ and $M_{\tilde{l}_{R}}$ for left-handed and right-handed sleptons, equality of the soft squark masses between the first and second generations, $M_{\tilde{q}_{1,2}}$, while the mass of the third generation squarks is kept as an independent free parameter $M_{\tilde{q}_{3}}$. We allow for only one non-zero trilinear coupling, $A_{t}$. The gaugino masses $M_{1}, M_{2}$ and $M_{3}$ are free parameters as well. In particular this allows to have $M_{1} \ll M_{2}$, implying a light neutralino much below the EW scale. The ratio of the doublet Higgs VEV's $\tan \beta$, the Higgs bilinear term, $\mu$, and the pseudoscalar mass $M_{A}$ are the remaining free parameters. This reduced set of parameters as compared to the pMSSM with 19 parameters captures the main feature of the light neutralino dark matter (DM) and allows in particular to explore thoroughly the region where the Higgs decays invisibly. However assuming a common third generation squark could have some influence on the Higgs predictions. Indeed the stops tend to be rather heavy because of the constraints from flavor physics on the sbottom sector thus limiting the impact of the stop sector on the loop-induced Higgs decays. For other studies and global fits of the MSSM with 19 free parameters see [51 54].

\section{$2.1 \quad$ Scanning method}

In order to thoroughly scan the parameter space we used a Markov Chain Monte-Carlo (MCMC), first presented in [55], the data set is the same one used in [56]. The code consists on a Metropolis-Hastings algorithm, and is based on micrOMEGAs2.4 [57 59] for the computation of all observables. The supersymmetric spectra are calculated with SuSpect [60].

Each point is generated by making a random step with a normal variation from the previous point in each dimension. Then, we compute its total prior $\mathcal{P}$, total likelihood $L$ and total weight $\mathcal{Q}=\mathcal{P} \times L$. It is kept with a probability $\operatorname{Min}\left(1, \mathcal{Q}^{\prime} / \mathcal{Q}\right)$, where $\mathcal{Q}^{\prime}$ is the total weight of the point being tested and $\mathcal{Q}$ is that of the source point. If the evaluated point is not kept, then a new point is generated from the last accepted point. Thus, the parameter space is scanned via a random walk by iterating this procedure.

The priors we impose are: a set of parameters has to lie within the boundaries of the parameter space given by Table 1, while a physical solution of the spectrum calculator and a neutralino LSP are required. Regarding likelihoods, these are displayed in Table I of [55]. We include limits on B physics observables, on the anomalous magnetic moment of the muon $(g-2)_{\mu}$, on the Higgs and sparticles masses obtained from LEP and the corrections to the $\rho$ parameter. In the case of the limits on the Higgs mass we used the SUSY-HIT [61] and the HiggsBounds packages $[62,63$ as in [50] .The HiggsBounds version used (3.1.3) include LEP and Tevatron results as well as first LHC results, while more recent results from CMS presented in 64, 65] were added a posteriori. Notice that we take the WMAP measurement on the dark matter relic density as a strict upper limit on the LSP relic density -obtained via the usual freeze out mechanism-, however, we allow the neutralino to have a relic density as low as $10 \%$ of the measured value. Indeed, the LSP could be only a fraction of the dark component, the rest corresponding to other dark particles or to a modified theory of gravity. For more details see 55 and [56].

The scans we performed in this study were aimed to give a general determination of the different configurations with neutralino masses at the weak scale and below. However, as it 
was shown in [50,55], it is difficult to find light $(\lesssim 30 \mathrm{GeV})$ neutralinos with a random walk: the probability of falling in these regions that require fine-tuning is rather small. Indeed, in the MSSM, the neutralino LSP has to annihilate via the exchange of either rather light Higgs bosons, scenarios that are heavily constrained by the Tevatron experiments as well as by CMS, or light sleptons, particularly of staus with masses close to the LEP lower bound of $81.9 \mathrm{GeV}[8]$.

Hence we used two different techniques to trigger the chains that scanned the parameter spaces. On one hand we let part of the chains start randomly, i.e. look randomly for a starting point with $\mathcal{Q} \neq 0$. On the other hand we used the previous knowledge of fine-tuned regions explored in [50,55 to set fixed starting points for the rest of the chains, in order to force the random walk to yield at least a few points in such regions.

A summary of the characteristics of the runs we present is given in Table 1 , for more information see [56].

\begin{tabular}{|c|c|c|c|}
\hline Parameter & Minimum & Maximum & Tolerance \\
\hline$M_{1}$ & 1 & 1000 & 3 \\
$M_{2}$ & 100 & 2000 & 30 \\
$M_{3}$ & 500 & 6500 & 10 \\
$\mu$ & 0.5 & 1000 & 0.1 \\
$\tan \beta$ & 1 & 75 & 0.01 \\
$M_{A}$ & 1 & 2000 & 4 \\
$A_{t}$ & -3000 & 3000 & 100 \\
$M_{\tilde{l}_{R}}$ & 70 & 2000 & 15 \\
$M_{\tilde{l}_{L}}$ & 70 & 2000 & 15 \\
$M_{\tilde{q}_{1,2}}$ & 300 & 2000 & 14 \\
$M_{\tilde{q}_{3}}$ & 300 & 2000 & 14 \\
\hline
\end{tabular}

Table 1: Intervals of free parameters used for the MSSM (GeV units).

\subsection{Astrophysical constraints}

Next we briefly describe additional constraints imposed only after exploring the parameter space with the MCMC. These constraints arise from two types of dark matter observables. First we consider the limit from the spin independent cross section for neutralino scattering on nucleons obtained by XENON100 [47. In the MSSM, the cross section for neutralino scattering is computed with micrOMEGAs and depends on the quark content of the nucleon as well as on the local dark matter density. To set the quark coefficients we use $\sigma_{\pi N}=45 \mathrm{MeV}$ and $\sigma_{0}=40 \mathrm{MeV}$. These values lead to rather conservative astroparticle physics bounds although recent lattice QCD results [66] indicate that the s-quark content could be smaller than previously thought, leading to a further $20 \%$ suppression of the spin independent cross sections. Since we allow the neutralino to be only a fraction of the dark matter component, we rescale the nominal value for the DM density, $\rho=0.3$, by the fraction of the neutralino relic density to the measured relic density. Thus we have the same rescaling in all astrophysical systems. Second we consider the limit on the photon flux resulting from neutralino pair annihilation in dwarf spheroidal galaxies extracted from Fermi-LAT data [48. The procedure followed was presented in [67] and assumes a NFW profile [68]. The predictions for both the photon flux and the direct detection spin inde- 
pendent rate are presented and discussed in [50] and [56, 69]. Basically, the limit from dSph's constrain the light $(<20 \mathrm{GeV})$ neutralino case, whether or not it is associated with a light pseudoscalar, while direct detection limits constrain the light neutralino/light pseudoscalar region as well as regions where the LSP has a large higgsino fraction. These results will be presented and discussed in a forthcoming publication [56].

\subsection{Collider constraints}

Since performing the parameter space exploration, new limits from the LHC were announced. The important limits on the Higgs sector are the ones for $h \rightarrow \tau \tau$ which constrain the low $M_{A^{-}}$ large $\tan \beta$ region of parameter space. Furthermore the new upper limit on $B R\left(B_{s} \rightarrow \mu^{+} \mu^{-}\right)<$ $1.08 \times 10^{-8}[70]$ also constrains the low $M_{A}$ region. We have imposed both these constraints a posteriori. The impact of the additional constraints from LHC is displayed in Fig. 1 in the $\tan \beta-M_{A}$ plane and in the $B R\left(B_{s} \rightarrow \mu^{+} \mu^{-}\right)-M_{A}$ plane. The points excluded by either the Higgs search or $B R\left(B_{s} \rightarrow \mu^{+} \mu^{-}\right)$are coloured in yellow (light grey). One can see that $B R\left(B_{s} \rightarrow \mu^{+} \mu^{-}\right)$excludes points at lower values of $\tan \beta$ than the Higgs searches (yellow points that are below the Higgs exclusion line in Fig. 1 (left panel) while some points that satisfy the $B R\left(B_{s} \rightarrow \mu^{+} \mu^{-}\right)$limit are constrained by Higgs searches (Fig. 1, right panel). In this plot and all the following we will use the same color code: the points excluded by collider constraints are yellow, those excluded by either XENON100 or Fermi-LAT are red and allowed points are green (dark grey). Note that the scan extends to unnaturally large values of $\tan \beta$, however the special features in the observables we will discuss do not require a very large value of $\tan \beta$.
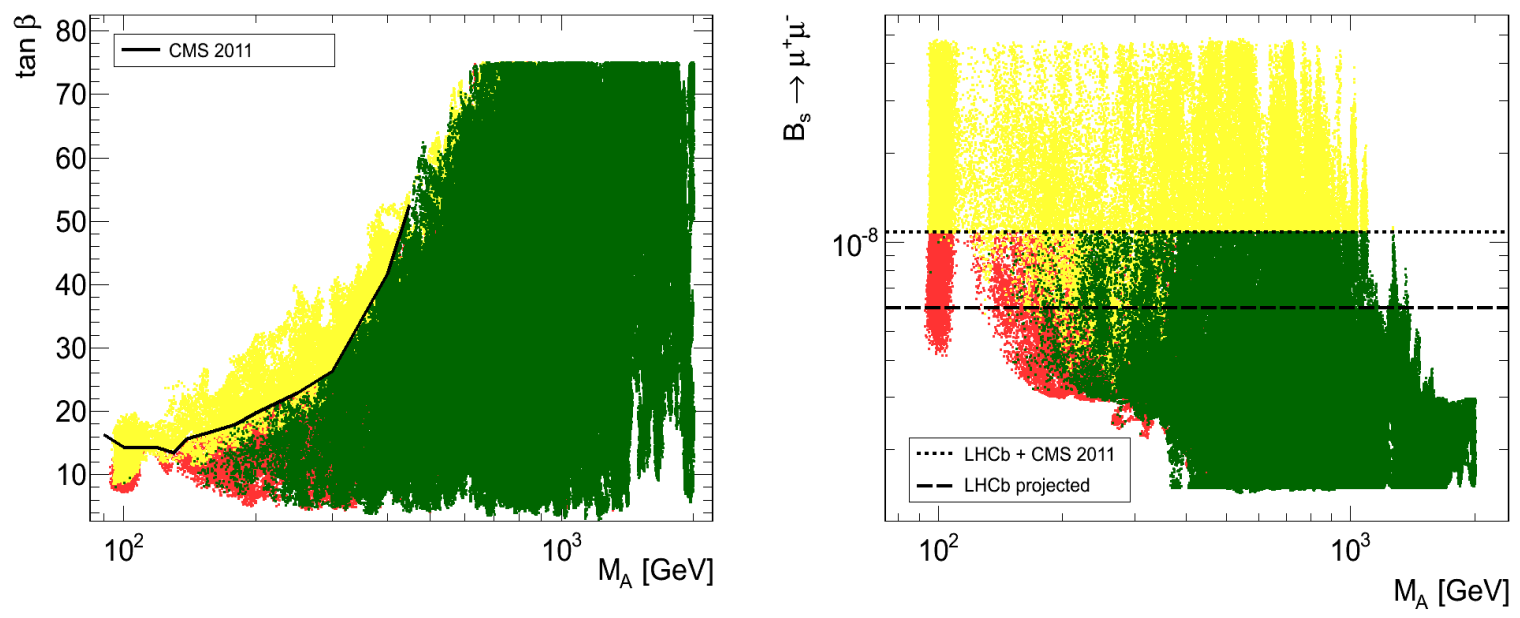

Figure 1: Left: Allowed points in the $\tan \beta$ vs. $M_{A}$ plane in green (dark grey). The exclusion limit from CMS [4] (full line) is also displayed. Right: Allowed points in the $B R\left(B_{s} \rightarrow \mu^{+} \mu^{-}\right)$ vs. $M_{A}$ plane. The exclusion limit from CMS and LHCb [70] (dotted line) is also displayed. In yellow, points excluded by collider constraints (Higgs and $B R\left(B_{s} \rightarrow \mu^{+} \mu^{-}\right)$), in red those excluded by astrophysical constraints (XENON100 and Fermi-LAT).

\section{Higgs observables}

The mass of the light Higgs in the MSSM scenarios that we consider is always below $130 \mathrm{GeV}$. Thus the most important detection channel is in the two-photon channel. In this mass range, the light Higgs is predominantly produced in gluon fusion. We define the ratio of the production 
times branching ratio of the MSSM to the SM,

$$
R_{g g \gamma \gamma}=\frac{\sigma(g g \rightarrow h)_{M S S M} B R(h \rightarrow \gamma \gamma)_{M S S M}}{\sigma(g g \rightarrow h)_{S M} B R(h \rightarrow \gamma \gamma)_{S M}}
$$

Note that $\sigma(g g \rightarrow h)$ is taken to be proportional to $\Gamma(h \rightarrow g g)$ even though QCD corrections are different for the two processes. We assume that the effect of QCD corrections cancels out when taking the ratio of the MSSM to the SM value. Both the MSSM and SM partial widths for $h \rightarrow g g, \gamma \gamma$ are computed with HDECAY 61].

Another search channel that has been used to set powerful limits on the MSSM Higgs sector is the $\tau \tau$ channel. Here the production is either through gluon fusion, from b-quarks, or $W W$ fusion. Thus we define three quantities for the ratio of the production times branching ratio of the MSSM to the SM

$$
\begin{gathered}
R_{g g \tau \tau}=\frac{\sigma(g g \rightarrow h)_{M S S M} B R(h \rightarrow \tau \bar{\tau})_{M S S M}}{\sigma(g g \rightarrow h)_{S M} B R(h \rightarrow \tau \bar{\tau})_{S M}} \quad R_{b b \tau \bar{\tau}}=\frac{\sigma(b b \rightarrow h)_{M S S M} B R(h \rightarrow \tau \bar{\tau})_{M S S M}}{\sigma(b b \rightarrow h)_{S M} B R(h \rightarrow \tau \bar{\tau})_{S M}} \\
R_{W W \tau \tau}=\frac{\sigma(W W \rightarrow h)_{M S S M} B R(h \rightarrow \tau \bar{\tau})_{M S S M}}{\sigma(W W \rightarrow h)_{S M} B R(h \rightarrow \tau \bar{\tau})_{S M}}
\end{gathered}
$$

Note that the latter is relevant for either WW fusion or associated W production.

Another search channel is the production of a Higgs in association with a gauge boson with the Higgs decaying into the $b \bar{b}$ final state. Similarly we define the ratio of the production times branching ratio of the MSSM to the SM

$$
R_{W W b b}=\frac{\sigma(W W \rightarrow h)_{M S S M} B R(h \rightarrow b \bar{b})_{M S S M}}{\sigma(W W \rightarrow h)_{S M} B R(h \rightarrow b \bar{b})_{S M}}
$$

Before showing our predictions for the observables just mentioned, we summarize the expectations for the Higgs couplings to SM particles in the MSSM. We define $R_{X X h}$ as the ratio of the Higgs coupling to a pair of SM particles $(X)$ in the MSSM to the same coupling in the SM. The $g g h$ coupling is dominated by the top quark but can also receive a large contribution from the third generation squarks, in particular from the stop sector. The coupling $h \rightarrow \gamma \gamma$ is dominated by the contribution from the $\mathrm{W}$ bosons with a contribution from top quarks almost an order of magnitude smaller. The two contributions have opposite signs. Supersymmetric contributions arise from the chargino and stop sector. Thus both the production and the decay can be significantly different from the SM. There are three mechanisms that can induce large corrections to some of the Higgs couplings and branching ratios: a light pseudoscalar, light supersymmetric particles in the loop and light LSP's.

When $M_{A}$ is light, that is in the non-decoupling case, the tree-level couplings of the Higgs to SM particles can show large deviations as compared to the SM case. For example, $R_{W W h}=$ $\sin (\alpha-\beta)$ and $R_{t t h}=\cos \alpha / \sin \beta$ where $\alpha$ is the Higgs mixing angle, are smaller than one only for low values of $M_{A}$ while $R_{b b h}=R_{\tau \tau h}=\sin \alpha / \cos \beta$ are enhanced especially at large values of $\tan \beta$ as shown in [5, 10]. Furthermore corrections to the $h b b$ vertex arise from higher order effects, in particular the $\Delta M_{b}$ correction can lead to a much enhanced $h b b$ coupling at large values of $\tan \beta[5]$. Because the $b b$ mode is the dominant decay channel of the light Higgs, in this case the total width of the Higgs becomes much larger in the MSSM. This means that the branching fraction in other modes, such as $B R(h \rightarrow \gamma \gamma)$ can be strongly suppressed even when the $h \gamma \gamma$ coupling is itself SM-like. It is also possible that $\Delta M_{b}$ corrections lead to a suppressed $h b b$ coupling in which case the branching fractions into all other modes could be enhanced. However this requires large values of $\tan \beta$, low value of $m_{A}$ as well as large values of $\mu$ [5], we did not find any such configurations in our MCMC analysis. 
The loop-induced Higgs couplings can also deviate from their SM value because of the contributions of the supersymmetric particles in the loop. The largest effect is from the stop sector [9]. The light stop interferes constructively with top when there is no mixing in the stop sector while interference is destructive in the large mixing case. In the first case $h g g$ will increase while in the latter it will decrease and a contrario the $h \gamma \gamma$ couplings. Since we have imposed an universal mass for the third generation, the mixing in the stop sector is large unless $A_{t}-\mu / \tan \beta \approx 0$ so we expect a decrease in the Higgs production via gluon fusion, that cannot be compensated by the more modest increase of the two-photon width. Note that the largest corrections are expected for light stops, while in our scans the stops are above $400 \mathrm{GeV}$ and typically above the $\mathrm{TeV}$ scale. The charginos can also affect the $h \gamma \gamma$ coupling, the contribution of charginos was shown to be at most of the order of $15 \%$ [16.

We will not consider the $W W$ decay mode of the Higgs, although the decay into virtual W's contribute to the total significance for the SM Higgs exclusion in the upper range of the MSSM light Higgs mass, it is much less important than the $\gamma \gamma$ mode. Furthermore, as discussed above we expect the $W W h$ coupling to be either SM-like or suppressed at low values of $M_{A}$, thus even lessening the importance of this channel.

\section{Results}

After performing the MCMC analysis and imposing the constraints described in the previous section, we examine the predictions for the light Higgs of the model.

\subsection{The invisible Higgs}

A large branching fraction of the Higgs into invisible particles will affect all Higgs observables in the MSSM. Apart form the obvious kinematic condition on the LSP mass, $M_{\tilde{\chi}_{1}^{0}}<M_{h} / 2$, a large partial width into invisible requires a significant coupling of the LSP to the Higgs. This means that the light neutralino which is predominantly bino must have also a higgsino component. Thus $\mu$ cannot be large. Since $\mu>100 \mathrm{GeV}$ because of the LEP constraints on charginos, this condition implies that for very light neutralinos, say below $20 \mathrm{GeV}$, the invisible width never exceeds $20-30 \%$. Indeed such a light neutralino cannot have a large higgsino component since $\mu \gg M_{1}$, see Fig. 2, left panel. For heavier neutralino LSP's the invisible width can reach $80 \%$. Note that when $M_{\tilde{\chi}_{1}^{0}} \approx M_{Z} / 2$ there is a dip in the invisible width - it does not exceed $50 \%$. This is because the coupling of the LSP to the $\mathrm{Z}$ must be somewhat suppressed for the annihilation of the LSP via Z-exchange not to be enhanced by the resonance effect, thus avoiding a too small value for the relic density. The $\tilde{\chi}_{1}^{0} \tilde{\chi}_{1}^{0} Z$ coupling also depends on the higgsino component of the LSP hence $\operatorname{BR}(h \rightarrow$ inv $)$ is suppressed at the same time. Note that a light LSP will also contribute to the invisible width of the Z. However, this constraint is taken into account in the numerical analysis.

There is a strong correlation between the mass of the lightest chargino and the invisible width. Indeed the small value for $\mu$ needed for the invisible Higgs drives the mass of the lightest chargino. We find that when the invisible width of the Higgs is larger than $20 \%$, then the chargino is lighter than $200 \mathrm{GeV}$, see Fig. 2, right panel. Furthermore since these points correspond in general to $\mu<M_{2}$, the chargino will be largely higgsino and the second and third neutralino will also have a mass of the same order.

In Fig. 3, we display $\mathrm{BR}(h \rightarrow$ inv $)$ as a function of the pseudoscalar mass. The invisible branching ratio is never very large for light pseudoscalar. This is because, as mentioned in section 3 , there is a large increase in the $h \rightarrow \bar{b} b$ partial width hence also in the total width. Although there is no direct correlation between the invisible width and $M_{A}$ - in the decoupling limit any value of the invisible width can be found - this figure is included to facilitate a com- 

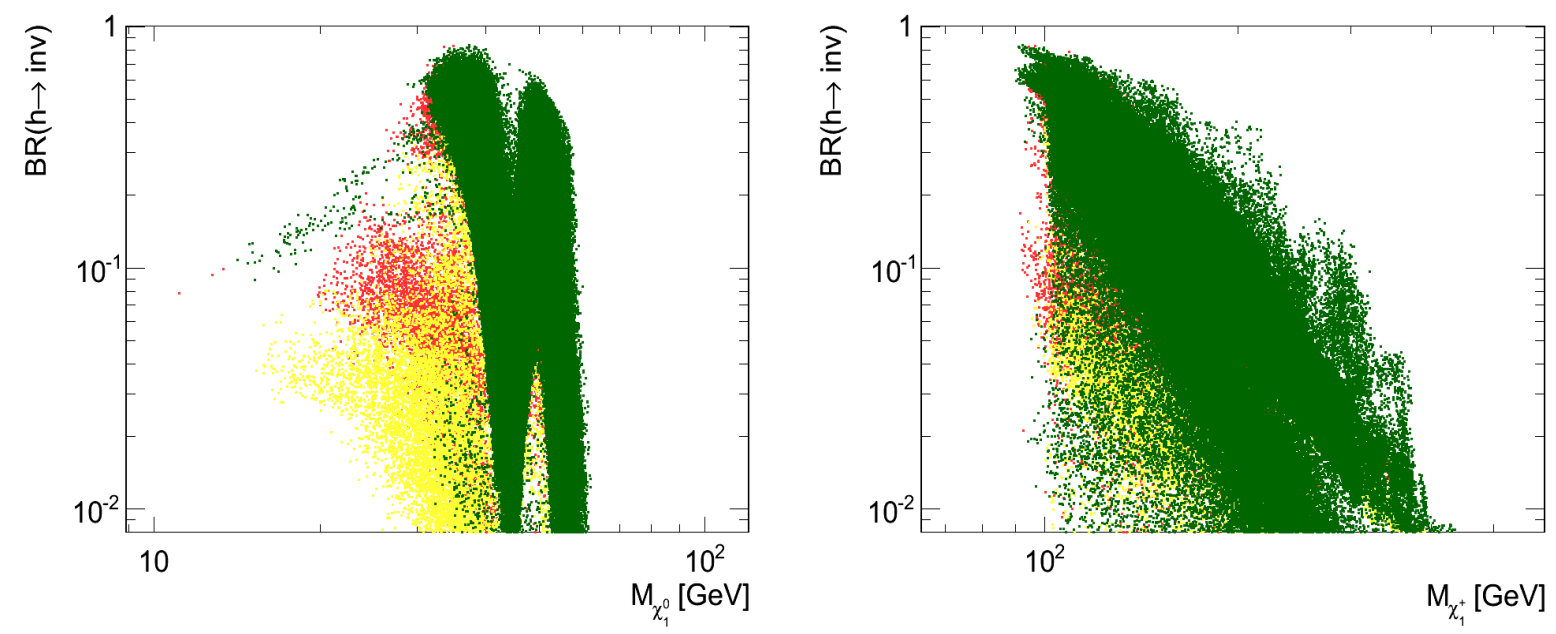

Figure 2: $\mathrm{BR}\left(h \rightarrow\right.$ inv) as a function of the LSP mass (left) and $M_{\tilde{\chi}+}$ (right), same color code as Fig. 1 .

parison with other Higgs observables which strongly depend on $M_{A}$. Note that a large invisible width can be found even for $M_{A}>1 \mathrm{TeV}$.

The Higgs can also decay into sneutrinos. These further decay into neutralinos thus contributing to the invisible width of the Higgs. This channel is usually not accessible kinematically since to have $M_{\tilde{\nu}}<M_{h} / 2$ requires that the LH soft mass for sleptons be below roughly $100 \mathrm{GeV}$. The charged sleptons are then strongly constrained by LEP. Only a few points where the charged sleptons are just above the LEP limit are allowed and have a sneutrino light enough for a dominant decay of the Higgs is into sneutrinos. In the numerical analysis we have included both the neutralino and sneutrino mode in the invisible width though the LSP is always a neutralino.

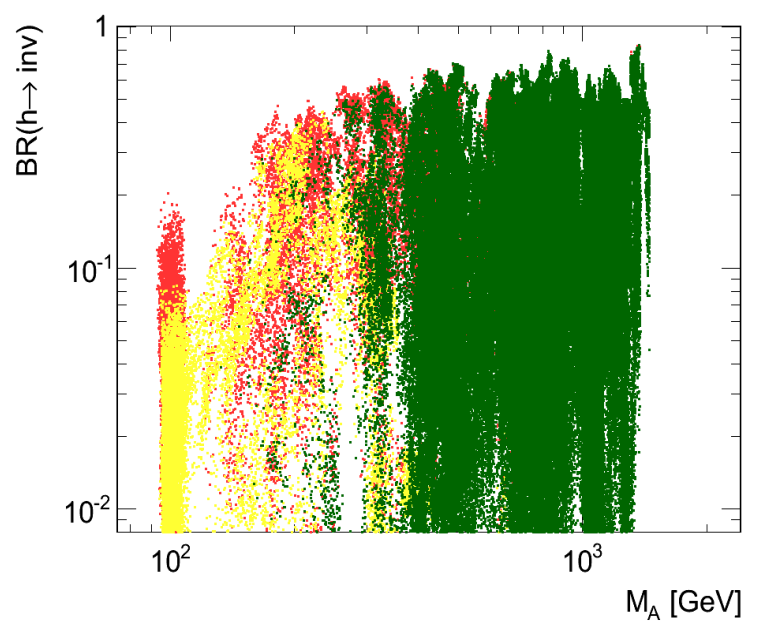

Figure 3: $\operatorname{BR}(h \rightarrow$ inv $)$ as a function of $M_{A}$. Same color code as Fig. 1 .

\section{$4.2 \quad g g \rightarrow h \rightarrow \gamma \gamma$}

The predictions for $R_{g g \gamma \gamma}$ as a function of the pseudoscalar mass are displayed in Fig. 4, left panel. The MSSM Higgs signal is expected to be at most as large as that of the SM Higgs. The observable $R_{g g \gamma \gamma}$ drops dramatically when $M_{A}$ is below about $200 \mathrm{GeV}$, as expected from 
the previous discussion. The upper limit on $R_{g g \gamma \gamma}$ is only 0.3(0.6) when $M_{A}=200(300) \mathrm{GeV}$. Although many of the points with a light pseudoscalar are already constrained by either FermiLAT, XENON100 (points in red), or latest Higgs searches or the new upper limit on $B R\left(B_{s} \rightarrow\right.$ $\mu^{+} \mu^{-}$) (points in yellow ), some points with $R_{g g \gamma \gamma} \approx 0.01$ and $M_{A} \approx 100 \mathrm{GeV}$ are still allowed. These points lie in the small window left by LEP constraints when all Higgses are roughly of the same mass. Furthermore there are still allowed points where the Higgs signal is only $10 \%$ of its SM value. Since these are associated with a light $M_{A}$, they can be probed further in searches for the neutral and charged component of the heavy Higgs doublet in CMS and ATLAS and/or a more precise measurement of $B R\left(B_{s} \rightarrow \mu^{+} \mu^{-}\right)$. Note that one needs to explore values of $\tan \beta<10$ to be able to probe these points.

When $M_{A}>400 \mathrm{GeV}$ and one is in the decoupling limit the suppression in $R_{g g \gamma \gamma}$ is usually more modest. Nevertheless it is still possible to have $R_{g g \gamma \gamma}$ as low as 0.2 even when $M_{A}$ is at the TeV scale. This occurs when the LSP is lighter than $M_{h} / 2$ and the invisible width of the Higgs is large. Note that there are a few points at large values of $M_{A}$ where $R \approx 0.01$ these all correspond to points where the LH soft mass for sleptons is below $100 \mathrm{GeV}$, the charged sleptons are just above the LEP exclusion and the sneutrino is light enough that the dominant decay of the Higgs is into sneutrinos. Other suppression of $R_{g g \gamma \gamma}$ arises from the stop sector. The effect is usually below $30 \%$, although for stop masses below $500 \mathrm{GeV}$ we found points where $R_{g g \gamma \gamma}$ can drop as low as 0.4 .

The predictions for $R_{g g \gamma \gamma}$ as a function of the light Higgs mass show that $R_{g g \gamma \gamma}$ has little dependence on the scalar mass provided it lies in the $110-130 \mathrm{GeV}$ range. However it is important to note that for a Higgs in the narrow mass range $110-114 \mathrm{GeV}$, we always find $R_{g g \gamma \gamma}<0.8$, because these values of $M_{h}$ are found only in the non-decoupling limit. Finally as explained above, the few allowed points around $M_{h}=100 \mathrm{GeV}$ feature a large drop in the Higgs two-photon signal.
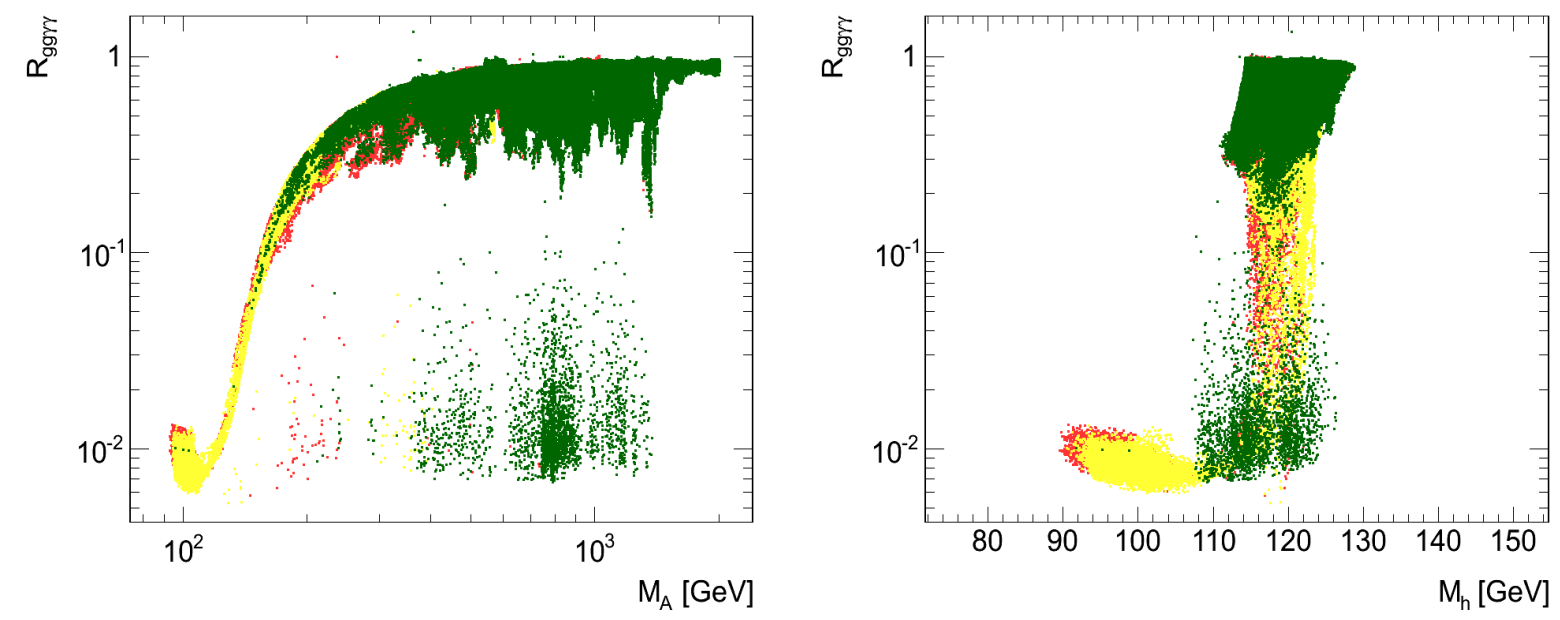

Figure 4: $R_{g g \gamma \gamma}$ as a function of the pseudoscalar mass (left) and light scalar mass (right) for allowed points. Same color code as Fig. 1

\section{3 $h \rightarrow f \bar{f}$}

Higgs searches are performed for two different final states, $b b$ and $\tau \tau$. First consider associated $W h$ production with the decay $h \rightarrow b \bar{b}$. In the decoupling limit, both the coupling $h W W$ and $h b b$ are near their SM value, thus we find that $R_{W W b b} \approx 1$ when $M_{A}>400 \mathrm{GeV}$ except for the points with a large invisible width. Since $b \bar{b}$ is the dominant decay mode of the Higgs in 
both the MSSM and the SM, there can only be a modest enhancement of the branching fraction even when the coupling is much enhanced. The largest enhancement of $B R(h \rightarrow b \bar{b})$ is found when $M_{A}<400 \mathrm{GeV}$. As mentioned in section 3, this leads to a value $R_{W W b b}$ that can reach 1.2 when the coupling $h W W$ is close to the SM value,see Fig. 5. However, when $M_{A}$ is lighter than $200 \mathrm{GeV}$, the $W W h$ coupling is strongly suppressed and $R_{W W b b}$ can drop to values as low as 0.1 .
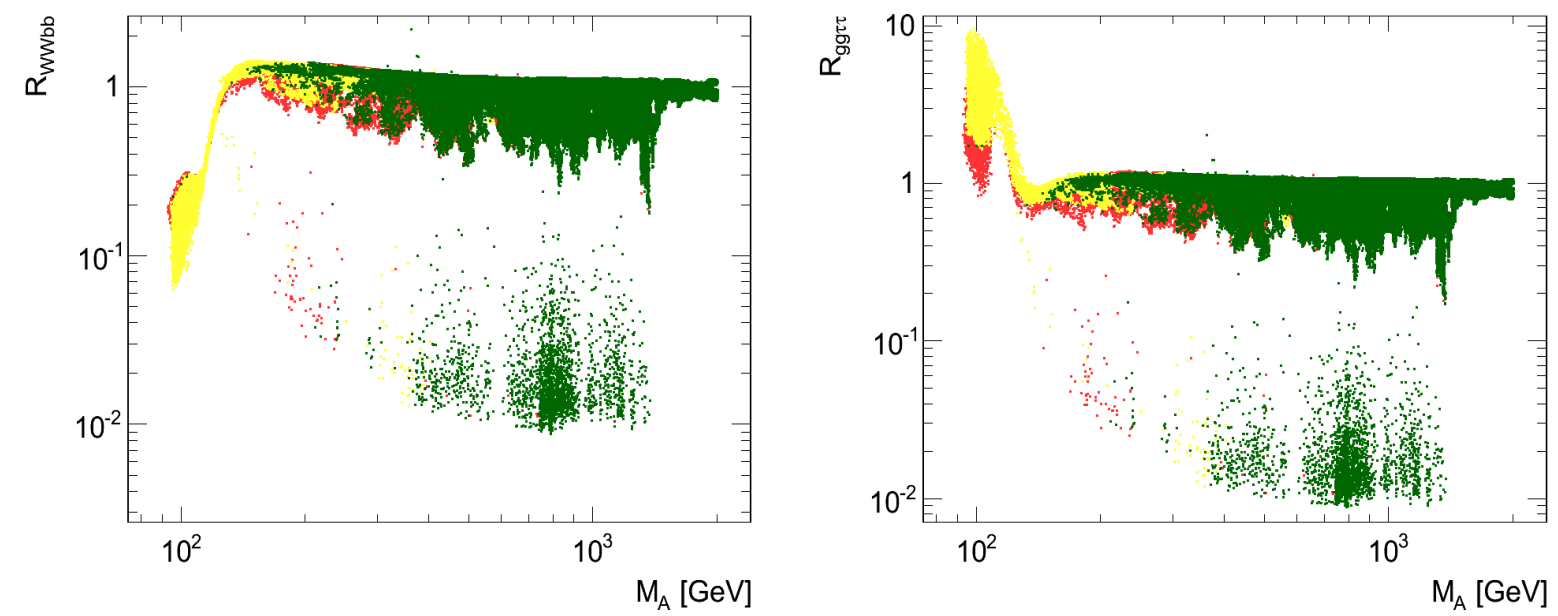

Figure 5: $R_{W W b b}$ (left) and $R_{g g \tau \tau}$ (right) as a function of the pseudoscalar mass for allowed points. Same color code as Fig. 1

Next we consider the signal in $h \rightarrow \tau \bar{\tau}$ treating separately three production processes. The dominant production process is gluon fusion. We find that $R_{g g \tau \tau}$ can reach almost 10, but only for values of $M_{A}$ that are constrained from heavy Higgs, $B R\left(B_{s} \rightarrow \mu^{+} \mu^{-}\right)$searches and/or from astrophysical constraints, see Fig. 5 (right panel). The few allowed points around $M_{A} \approx 100 \mathrm{GeV}$ have $R_{g g \tau \tau} \approx 2$ due an increase of the $g g h$ coupling resulting from a much enhanced contribution of b quarks in the loop. Otherwise $R_{g g \tau \tau}$ does not deviate much from the SM (0.8-1.1) unless there is a large invisible width.

The second process with Higgs production via W fusion and decaying into $\tau \tau$ final states is driven by $R_{W W \tau \tau}$. This quantity behaves very much like $R_{W W b b}$ discussed above. Indeed, at tree-level the branching fraction into tau pairs is proportional to the $b \bar{b}$ branching. Supersymmetric radiative corrections can induce shifts in the $h b b$ coupling, however we have checked that for the set of allowed points, the $\tau \tau$ and $b b$ branching ratios are well correlated. The third process is Higgs production from $b$-quarks. It is for this channel that we find the most dramatic effect as compared to the SM expectations. Indeed for small values of $M_{A}$ the $h b b$ coupling can be increased, section 3 , thus leading to up to $R_{b b \tau \tau} \approx 100$, see Fig. 6. Such large enhancements are possible even for intermediate values of $\tan \beta$. In fact for very large values of $\tan \beta, R_{b b \tau \tau}$ is at most equal to the SM value, see right panel of Fig. 6. This is because $M_{A}$ is strongly constrained from LHC searches when $\tan \beta$ is large. Altogether the $b b \tau \tau$ channel is mostly enhanced as compared to the SM, apart from the points with a significant invisible width, although the deviation from the SM prediction is quite modest when $M_{A}>400 \mathrm{GeV}$, see Fig. 6. Note however that the SM production rate of the Higgs from b quarks is very small as compared with $\mathrm{W}$ and gluon fusion. 

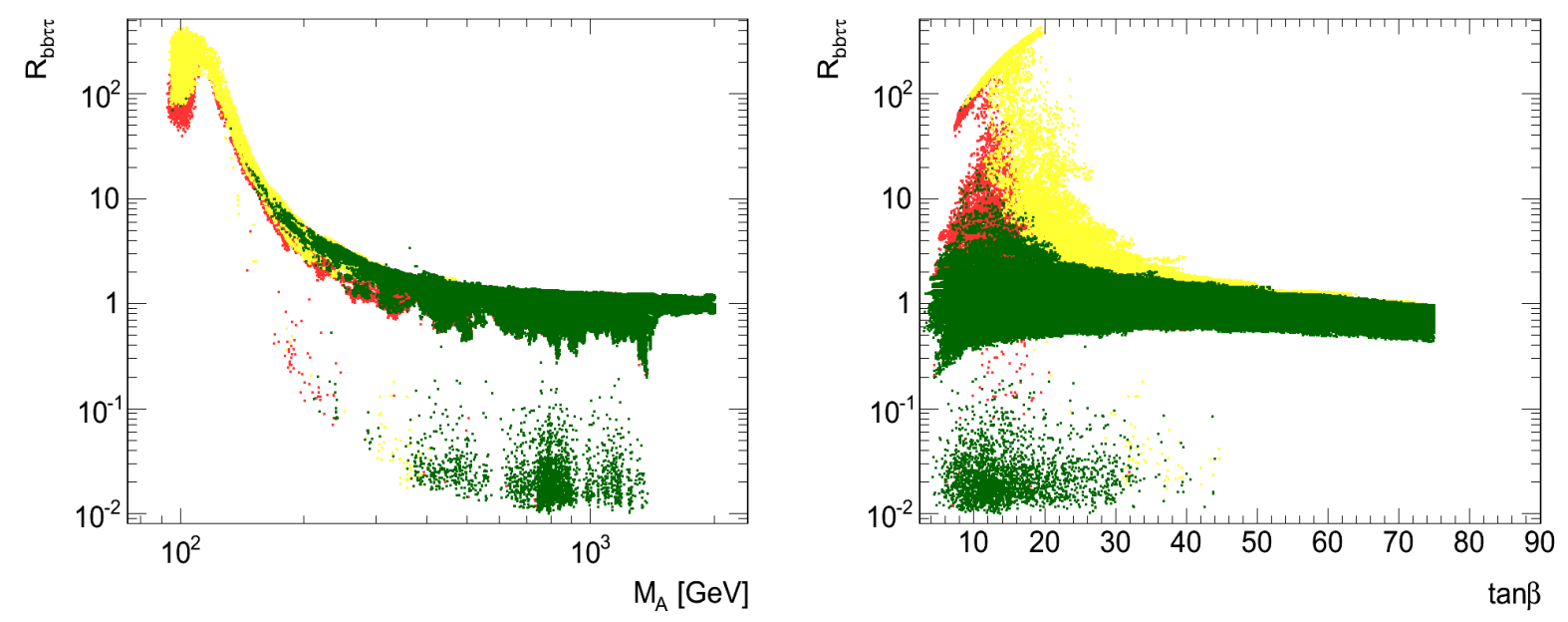

Figure 6: $R_{b b \tau \tau}$ as a function of the pseudoscalar mass (left) or $\tan \beta$ (right) mass for allowed points. Same color code as Fig. 1

\subsection{Correlation between Higgs search channels}

The correlation between the large invisible width and the suppressed $R_{g g \gamma \gamma}$ is displayed in Fig. 7, left panel. When $\operatorname{BR}(h \rightarrow$ inv $)$ is at the percent level, one sees a large allowed band where $R_{g g \gamma \gamma}>0.7$. As $\operatorname{BR}(h \rightarrow$ inv $)$ increases, the value of $R_{g g \gamma \gamma}$ drops to less than $20 \%$. To the left of this band there are a good number of points which have $R_{g g \gamma \gamma}$ between 0.3-0.7 even when the invisible width is small. These correspond to values of $M_{A}<300 \mathrm{GeV}$ where the $B R(h \rightarrow \gamma \gamma)$ is suppressed because of a large enhancement of the total width of the Higgs. Finally there are a number of scattered points with very suppressed $R_{g g \gamma \gamma}$ corresponding to the small values of $M_{A}$. Despite the fact that the production process is the same, there is no direct correlation between the channels $R_{g g \gamma \gamma}$ and $R_{g g \tau \tau}$ only when the Higgs decays invisibly, this is the branch where both channels are suppressed in Fig. 7. There are scenarios with a strong suppression in $R_{g g \gamma \gamma}$ where $R_{g g \tau \tau}$ is near or above 1. Again this is due to the large enhancement of the total width of the Higgs due to a large $h b b$ coupling. We therefore also find in this case $R_{W W b b}$ to be near 1 . Conversely there are cases where $R_{g g \gamma \gamma} \approx 1$ and $R_{g g \tau \tau}$ is suppressed, see Fig. 7. Note that these points should all be probed in the most sensitive channel, $g g \rightarrow h \rightarrow \gamma \gamma$, since a cross section at the SM level will be probed in the near future [3, 4]

To analyse the impact of suppressing the signal in one channel, we have selected the points with $R_{g g \gamma \gamma}<0.8$. We have also imposed $M_{A}>200 \mathrm{GeV}$ because we have already argued that the light pseudoscalar region would be probed further by the $H / A / h \rightarrow \tau \tau$ search. We find that when $\mathrm{BR}(h \rightarrow \mathrm{inv})$ is at the percent level, $M_{A}<500 \mathrm{GeV}$ and $R_{g g \tau \tau}, R_{W W \tau \tau}, R_{W W b b}$ are all greater than 1, in agreement with our argument that the reduction in $R_{g g \gamma \gamma}$ is largely due to the increase of $h b b$. Furthermore we also find that $R_{b b \tau \tau}$ is enhanced. When $\operatorname{BR}(h \rightarrow$ inv $)$ increases, all channels can be reduced. However it is only when the invisible width becomes large that new possibilities to probe the Higgs through the invisible mode can be used. Furthermore luminosities larger than $10 \mathrm{fb}^{-1}$ might be required [39,41,71].

\subsection{Spectrum of superparticles}

As we have seen in the previous section, there are points that fit all constraints and where one/all the Higgs discovery channels are suppressed. As we have discussed above, the suppression of some Higgs channel is often tied to the presence of not too heavy supersymmetric particles and/or pseudoscalar/charged Higgs. In such a case, direct searches for supersymmetric particles 

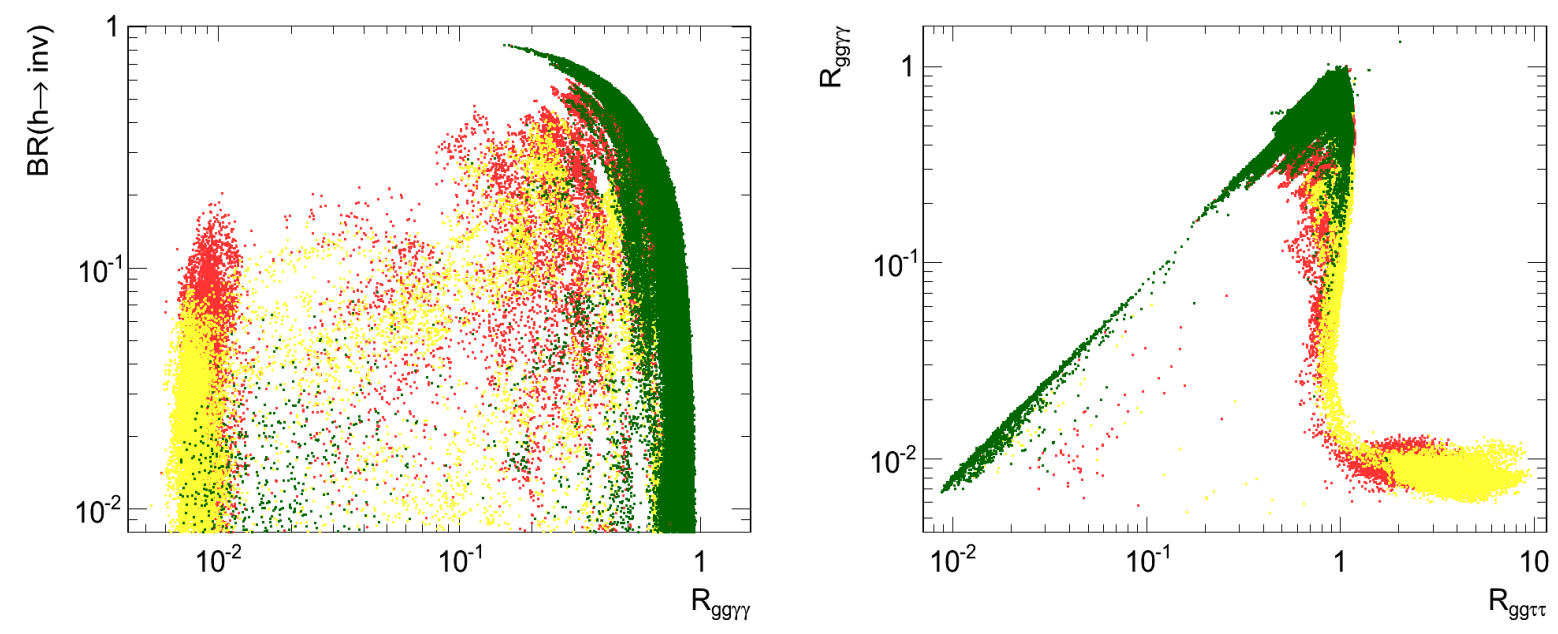

Figure 7: Allowed points in the plane $\mathrm{BR}(h \rightarrow \mathrm{inv})$ vs $R_{g g \gamma \gamma}(\mathrm{left})$ and $R_{g g \gamma \gamma}$ vs $R_{b b \tau \tau}$, same color code as Fig. 1 .

are important and complementary to the light Higgs searches. As an example, we consider the case where the main Higgs channel $R_{g g \gamma \gamma}<0.8$ and $M_{A}>200 \mathrm{GeV}$, and examine the impact on the supersymmetric spectrum. The distribution of the chargino mass is strongly shifted towards lower values $M_{\tilde{\chi}_{1}^{+}}<200 \mathrm{GeV}$. As argued above this is because the drop in $R_{g g \gamma \gamma}$ is mostly due to the invisible width which to be large requires a non-negligible higgsino content in the LSP, hence small values of $\mu$ and $M_{\tilde{\chi}_{1}^{+}}$. The slepton mass distribution is also shifted towards lower values. Even though the slepton mass does not in general directly enter the Higgs production and decay, selecting suppressed $R_{g g \gamma \gamma}$ means including all points with a large invisible width thus with a light bino LSP and a light chargino. The constraint from $(g-2)_{\mu}$ is then easily satisfied either with the neutralino smuon contribution which requires not too heavy smuons or from the chargino/sneutrino contribution which requires large values of $\tan \beta$ and light sneutrinos [72,73]. In both cases light sleptons are preferred. Note that a large invisible width of the Higgs into sneutrinos is associated with $M_{\tilde{l}} \approx 100 \mathrm{GeV}$. Finally the stop quark mass distribution spans the whole range of allowed values thus proving to be uncorrelated to this selection.

Searches for purely electroweak particles, charginos, neutralinos and sleptons will therefore offer a powerful and complementary probe of Supersymmetry in cases where the light Higgs is hidden. A more quantitative analysis of the potential of the LHC to probe these channels is kept for a future work.

\subsection{Benchmarks}

To illustrate the different possible scenarios for the main Higgs search channels that we have discussed in the previous section, we list a few benchmarks in Table 2. The first three benchmarks have a small invisible width, yet $R_{g g \gamma \gamma}<1$ while the last three have a large invisible width. Benchmark A is an example with $M_{A}<100 \mathrm{GeV}$ where the $h \rightarrow b \bar{b}$ partial width and hence the total width is strongly enhanced, leading to suppressed branching ratios for $h \rightarrow \gamma \gamma$. This scenario features squarks below $700 \mathrm{GeV}$ which are easily probed at the LHC. Benchmark B feature a heavier pseudoscalar, yet the $h b b$ coupling is enhanced, leading again to a suppressed $B R(h \rightarrow \gamma \gamma)$, furthermore supersymmetric particles reduce the $h g g$ vertex. Benchmark $\mathrm{C}$ is an example where the main effect comes from the stop loop in the $h g g$ vertex. Benchmark D and $\mathrm{E}$ are two examples where the Higgs decays invisibly, for two different pseudoscalar masses. Both cases feature a light chargino which is dominantly higgsino. Benchmark $\mathrm{F}$ is an example 

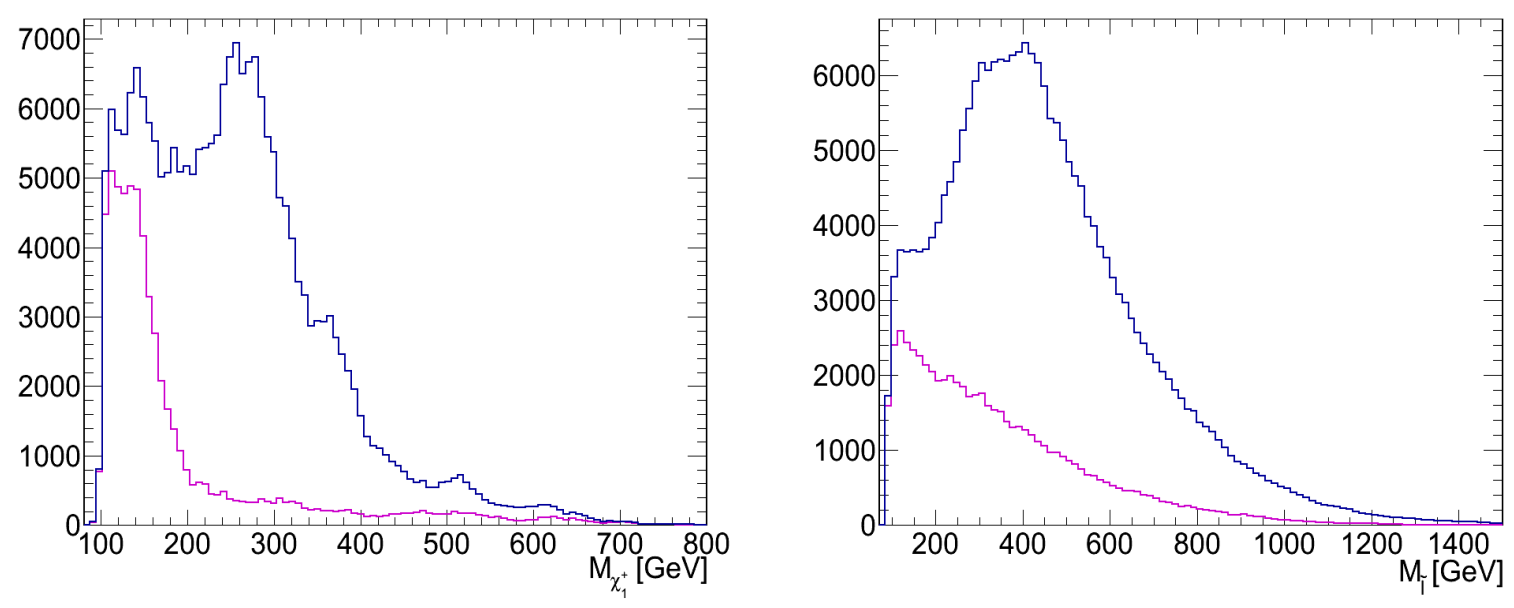

Figure 8: The distribution for the lightest chargino(left) and lightest slepton(right) mass for all allowed points (top,blue) and for the case where $R_{g g \gamma \gamma}<0.8$ and $M_{A}>200 \mathrm{GeV}$ (bottom,purple).

where the sneutrino is light, $M_{\tilde{\nu}}=44.4 \mathrm{GeV}$, the invisible Higgs is near $100 \%$ and all channels are suppressed. This scenario is best probed by slepton searches. The benchmarks illustrate explicitly the fact that the modification of the SM rate is not the same in each channel, even for a common final state. As the sensitivity of the Higgs searches improve, it would be useful to provide the results for each production times decay channel separately.

\section{Discussion}

We have displayed the predictions for several search channels. We briefly compare our results with the expectations from the LHC. The combined ATLAS and CMS limits for the $h \gamma \gamma$ channel exclude $\sigma / \sigma_{S M}<3.1$ in the mass range $100-121 \mathrm{GeV}$ and $\sigma / \sigma_{S M}<1.8$ in the $121-131 \mathrm{GeV}$ range. For the higher mass range, one expects to reach $\sigma / \sigma_{S M} \approx 1$ with the integrated luminosity $\mathcal{L}=5 \mathrm{fb}^{-1}$ collected in 2011 , assuming a naive rescaling of the current limit with $\sqrt{\mathcal{L}}$. This is compatible with the forecast of the CMS and ATLAS collaborations. A further increase in luminosity to $10 \mathrm{fb}^{-1}$ would allow to probe a significant fraction of the supersymmetric parameter space through the light Higgs search in $\gamma \gamma$, since it would cover roughly the case where $R_{g g \gamma \gamma}>$ $0.7-0.8$. To probe a Higgs below $120 \mathrm{GeV}$ will require at least $10 \mathrm{fb}^{-1}$ in the $h \gamma \gamma$ channel, and even more in the case $110 \mathrm{GeV}<M_{h}<114 \mathrm{GeV}$ since not only the exclusion is not as stringent but also the signal is expected to be suppressed as compared to the SM. A luminosity of $10 \mathrm{fb}^{-1}$ might be sufficient to start excluding the Higgs in the $\tau \tau$ channel if $M_{h}<125 \mathrm{GeV}$, especially when there is an enhancement over the SM expectation. In fact for $M_{h} \approx 120 \mathrm{GeV}$ both the $\gamma \gamma$ and $\tau \tau$ channels are competitive in the scenarios where the latter is enhanced. The case $110 \mathrm{GeV}<M_{h}<114 \mathrm{GeV}$ as well as the main channel will require a much higher sensitivity since a suppression occurs for the $\tau \tau$ and $b \bar{b}$ channels. A complete investigation of the MSSM light Higgs calls for a dedicated search for the invisible Higgs, as a large invisible width is allowed provided the neutralino LSP (or the sneutrino) is light.

A complete exploration of the parameter space of the MSSM is a daunting task. Nevertheless, even though we restricted our analysis to the MSSM with 11 parameters we think we have covered the essential features of the Higgs signals in different channels, especially concerning the difficult scenarios where the main channel is suppressed. In this study we have restricted ourselves to the case of common left and right stop masses, which automatically gives a large mixing among the 
third generation squarks, and have imposed soft squark masses above $500 \mathrm{GeV}$. If the mixing in the stop sector is small, $g g h$ will in fact increase as discussed in section 3 leading to a stronger signal in the most sensitive search channel. These scenarios will therefore be well covered by Higgs searches. We might nevertheless have missed some light stop scenarios with a large mixing which have a suppressed production rate in the $g g$ mode. These should be probed by direct searches for light stop quark at the LHC [10].

We have also restricted our analysis to $\Omega h^{2}>10 \% \Omega_{W M A P}$ for a neutralino LSP as the DM candidate. This means that we have excluded scenarios where the neutralino LSP has a large higgsino or wino component and constitutes a negligible component of the dark matter. The most significant effect of a small value of $\mu$, thus large higgsino component, implies a large invisible width of the Higgs when the neutralino LSP is light. This case was however explored thoroughly in our study since the light LSP has typically a large value of $\Omega h^{2}$. The value of $\mu$ can impact the loop induced couplings of the Higgs, however the effect is typically below the $20 \%$ level, similar to what we have found in the the scenarios considered in our analysis. Note that heavier LSP's with large higgsino component are also strongly constrained by direct detection searches. we have also checked that by restricting $\Omega h^{2}$ to be compatible with the value measured by WMAP does not affect significantly our predictions for the light Higgs production and decays.

In conclusion our analysis shows that when taking into account dark matter and flavour constraints in the MSSM, the light Higgs signal in the $\gamma \gamma$ channel is expected to be at most at the level of the SM Higgs. These results agree with another analysis in the case where the invisible width is small [52]. Strong suppression of the signal occur in two different cases: low $M_{A}$ or large invisible width. If such strong suppression occur, one must rely on alternate channels, such as the search for the heavy pseudoscalar, the charged Higgs or supersymmetric particles. A more modest suppression is associated with the effect of light supersymmetric particles. Observation of a SM-like Higgs signal in the mass range compatible with MSSM predictions would therefore strongly constrain the light pseudoscalar as well as light neutralino MSSM scenarios. Therefore new particles searches and Higgs searches provide complementary probes of supersymmetry.

\section{Acknowledgements}

We thank Guillaume Drieu La Rochelle, Fawzi Boudjema, Céline Boehm and Sabine Kraml for useful discussions. GB thanks the LPSC, Grenoble and RMG thanks the LAPTH for their hospitality. This work was supported in part by the GDRI-ACPP of CNRS. The work of AP was supported by the Russian foundation for Basic Research, grant RFBR-10-02-01443-a. RMG wishes to acknowledge the Department of Science and Technology of India, for financial support under the J.C. Bose Fellowship scheme under grant no. SR/S2/JCB-64/2007.

\section{References}

[1] ATLAS and CMS Collaboration, Combined Standard Model Higgs boson searches with up to 2.3 inverse femtobarns of pp collision data at sqrt(s)=7 TeV at the LHC, CMS PAS HIG-11-023, ATLAS-Conf-2011-157.

[2] CMS Collaboration, S. Chatrchyan et. al., Search for a Higgs boson decaying into two photons in the CMS detector, CMS PAS HIG-11-021.

[3] F. Gianotti (for ATLAS) and G. Tonelli (for CMS), talks given at the CERN seminar on update on the Standard Model Higgs searches, CERN, 13/12/2011;

https://indico. cern. ch/conferenceDisplay . py? conf $I d=164890$. 
[4] CMS Collaboration, S. Chatrchyan et. al., Search for Neutral Higgs Bosons Decaying to Tau Pairs in pp Collisions at sqrts=7 TeV, CMS PAS HIG-11-020.

[5] A. Djouadi, The anatomy of electro-weak symmetry breaking. II. The Higgs bosons in the minimal supersymmetric model, Phys.Rept. 459 (2008) 1-241, hep-ph/0503173.

[6] M. Drees, R. Godbole, and P. Roy, Theory and phenomenology of sparticles, World Scientific (2005).

[7] H. Baer and X. Tata, Weak scale supersymmetry: From superfields to scattering events, Cambridge, UK: Univ. Pr. (2006) 537 p.

[8] Particle Data Group Collaboration, K. Nakamura et. al., Review of particle physics, J.Phys.G G37 (2010) 075021.

[9] A. Djouadi, Squark effects on Higgs boson production and decay at the LHC, Phys.Lett. B435 (1998) 101-108, hep-ph/9806315.

[10] G. Belanger, F. Boudjema, and K. Sridhar, SUSY Higgs at the LHC: Large stop mixing effects and associated production, Nucl.Phys. B568 (2000) 3-39, hep-ph/9904348.

[11] M. S. Carena, S. Heinemeyer, C. Wagner, and G. Weiglein, Suggestions for benchmark scenarios for MSSM Higgs boson searches at hadron colliders, Eur.Phys.J. C26 (2003) 601-607, hep-ph/0202167.

[12] R. Kinnunen, S. Lehti, A. Nikitenko, and P. Salmi, On the discovery potential of the lightest MSSM Higgs boson at the LHC, J.Phys.G G31 (2005) 71-88, hep-ph/0503067.

[13] R. Dermisek and I. Low, Probing the Stop Sector and the Sanity of the MSSM with the Higgs Boson at the LHC, Phys.Rev. D77 (2008) 035012, hep-ph/0701235.

[14] I. Low and S. Shalgar, Implications of the Higgs Discovery in the MSSM Golden Region, JHEP 0904 (2009) 091, arXiv:0901.0266.

[15] A. Djouadi, V. Driesen, W. Hollik, and J. I. Illana, The Coupling of the lightest SUSY Higgs boson to two photons in the decoupling regime, Eur.Phys.J. C1 (1998) 149-162, hep-ph/9612362.

[16] G. Belanger, F. Boudjema, F. Donato, R. Godbole, and S. Rosier-Lees, SUSY Higgs at the LHC: Effects of light charginos and neutralinos, Nucl.Phys. B581 (2000) 3-33, hep-ph/0002039.

[17] U. Ellwanger, Enhanced di-photon Higgs signal in the Next-to-Minimal Supersymmetric Standard Model, Phys.Lett. B698 (2011) 293-296, arXiv:1012.1201.

[18] M. Carena, E. Ponton, and J. Zurita, BMSSM Higgs Bosons at the Tevatron and the LHC, Phys.Rev. D82 (2010) 055025, arXiv:1005.4887.

[19] M. Carena, E. Ponton, and J. Zurita, BMSSM Higgs Bosons at the 7 TeV LHC, arXiv:1111.2049.

[20] F. Boudjema and G. Drieu La Rochelle, arXiv:1112.1434 [hep-ph].

[21] G. Belanger, F. Boudjema, A. Cottrant, R. Godbole, and A. Semenov, The MSSM invisible Higgs in the light of dark matter and g-2, Phys.Lett. B519 (2001) 93-102, hep-ph/0106275. 
[22] M. Hirsch, J. Romao, J. Valle, and A. Villanova del Moral, Production and decays of supersymmetric Higgs bosons in spontaneously broken R-parity, Phys.Rev. D73 (2006) 055007, hep-ph/0512257.

[23] E. Accomando, A. Akeroyd, E. Akhmetzyanova, J. Albert, A. Alves, et. al., Workshop on CP Studies and Non-Standard Higgs Physics, hep-ph/0608079. Report of the Workshop on CP Studies and Non-standard Higgs Physics, CERN, Geneva, Switzerland, May 2004 Dec 2005.

[24] N. Arkani-Hamed, L. J. Hall, H. Murayama, D. Tucker-Smith, and N. Weiner, Small neutrino masses from supersymmetry breaking, Phys.Rev. D64 (2001) 115011, hep-ph/0006312.

[25] Z. Thomas, D. Tucker-Smith, and N. Weiner, Mixed Sneutrinos, Dark Matter and the CERN LHC, Phys.Rev. D77 (2008) 115015, arXiv:0712.4146.

[26] G. Belanger, M. Kakizaki, E. Park, S. Kraml, and A. Pukhov, Light mixed sneutrinos as thermal dark matter, JCAP 1011 (2010) 017, arXiv:1008.0580.

[27] U. Ellwanger, J. F. Gunion, and C. Hugonie, Difficult scenarios for NMSSM Higgs discovery at the LHC, JHEP 0507 (2005) 041, hep-ph/0503203.

[28] U. Ellwanger, C. Hugonie, and A. M. Teixeira, The Next-to-Minimal Supersymmetric Standard Model, Phys.Rept. 496 (2010) 1-77, arXiv:0910.1785.

[29] D. G. Cerdeno, J.-H. Huh, M. Peiro, and O. Seto, Very light right-handed sneutrino dark matter in the NMSSM, JCAP 1111 (2011) 027, arXiv:1108.0978.

[30] Y. Mambrini, Higgs searches and singlet scalar dark matter: when XENON100 meets the $L H C$, arXiv:1108.0671 [hep-ph].

[31] K. Ghosh, B. Mukhopadhyaya, and U. Sarkar, Signals of an invisibly decaying Higgs in a scalar dark matter scenario: a study for the Large Hadron Collider, Phys.Rev. D84 (2011) 015017, arXiv:1105.5837.

[32] S. Andreas, C. Arina, T. Hambye, F.-S. Ling, and M. H. G. Tytgat, A light scalar WIMP through the Higgs portal and CoGeNT, arXiv:1003.2595.

[33] O. Lebedev, H. M. Lee, and Y. Mambrini, Vector Higgs-portal dark matter and the invisible Higgs, arXiv:1111.4482

[34] G. F. Giudice, R. Rattazzi, and J. D. Wells, Graviscalars from higher dimensional metrics and curvature Higgs mixing, Nucl.Phys. B595 (2001) 250-276, hep-ph/0002178.

[35] M. Battaglia, D. Dominici, J. Gunion, and J. Wells, The Invisible Higgs decay width in the add model at the LHC, hep-ph/0402062.

[36] J. van der Bij and S. Dilcher, A Higher dimensional explanation of the excess of Higgs-like events at CERN LEP, Phys.Lett. B638 (2006) 234-238, hep-ph/0605008.

[37] J. van der Bij, The Minimal non-minimal standard model, Phys.Lett. B636 (2006) 56-59, hep-ph/0603082. In memory of Alfred Hill.

[38] K. Belotsky, D. Fargion, M. Khlopov, R. Konoplich and K. Shibaev, Invisible Higgs boson decay into massive neutrinos of fourth generation,' Phys. Rev. D 68 (2003) 054027 hep-ph/0210153. 
[39] O. J. Eboli and D. Zeppenfeld, Observing an invisible Higgs boson, Phys.Lett. B495 (2000) 147-154, hep-ph/0009158.

[40] H. Davoudiasl, T. Han, and H. E. Logan, Discovering an invisibly decaying Higgs at hadron colliders, Phys.Rev. D71 (2005) 115007, hep-ph/0412269].

[41] R. Godbole, M. Guchait, K. Mazumdar, S. Moretti, and D. Roy, Search for 'invisible' Higgs signals at LHC via associated production with gauge bosons, Phys.Lett. B571 (2003) 184-192, hep-ph/0304137].

[42] D. Cavalli, A. Djouadi, K. Jakobs, A. Nikitenko, M. Spira, et. al., The Higgs working group: Summary report, hep-ph/0203056.

[43] Higgs Working Group Collaboration, K. Assamagan et. al., The Higgs working group: Summary report 2003, hep-ph/0406152.

[44] C. Buttar, S. Dittmaier, V. Drollinger, S. Frixione, A. Nikitenko, et. al., Les houches physics at TeV colliders 2005, standard model and Higgs working group: Summary report, hep-ph/0604120.

[45] I. Low, P. Schwaller, G. Shaughnessy, and C. E. Wagner, The dark side of the Higgs boson, arXiv:1110.4405.

[46] E. Boos, S. Demidov, and D. Gorbunov, Invisible Higgs in weak bosons associative production with heavy quarks at LHC: probing mass and width, Int.J.Mod.Phys. A26 (2011) 3201-3218, arXiv:1010.5373].

[47] XENON100 Collaboration, E. Aprile et. al., Dark Matter Results from 100 Live Days of XENON100 Data, Phys.Rev.Lett. 107 (2011) 131302, arXiv:1104.2549.

[48] A. Abdo, M. Ackermann, M. Ajello, W. Atwood, L. Baldini, et. al., Observations of Milky Way Dwarf Spheroidal galaxies with the Fermi-LAT detector and constraints on Dark Matter models, Astrophys.J. 712 (2010) 147-158, arXiv:1001.4531.

[49] T. Falk, K. A. Olive, and M. Srednicki, Heavy sneutrinos as dark matter, Phys.Lett. B339 (1994) 248-251, hep-ph/9409270.

[50] D. A. Vasquez, G. Belanger, and C. Boehm, Revisiting light neutralino scenarios in the MSSM, Phys. Rev. D 84, 095015 (2011) arXiv:1108.1338.

[51] S. Sekmen, S. Kraml, J. Lykken, F. Moortgat, S. Padhi, et. al., Interpreting LHC SUSY searches in the phenomenological MSSM, arXiv:1109.5119.

[52] A. Arbey, M. Battaglia, and F. Mahmoudi, Implications of LHC Searches on SUSY Particle Spectra: The pMSSM Phase Space with Neutralino Dark Matter, arXiv: 1110.3726 .

[53] C. F. Berger, J. S. Gainer, J. L. Hewett, and T. G. Rizzo, Supersymmetry Without Prejudice, JHEP 0902 (2009) 023, arXiv:0812.0980.

[54] S. S. AbdusSalam, B. C. Allanach, F. Quevedo, F. Feroz, and M. Hobson, Fitting the Phenomenological MSSM, Phys.Rev. D81 (2010) 095012, arXiv:0904.2548.

[55] D. A. Vasquez, G. Belanger, C. Boehm, A. Pukhov, and J. Silk, Can neutralinos in the MSSM and NMSSM scenarios still be light?, Phys.Rev. D82 (2010) 115027, arXiv:1009.4380. 
[56] D. Albornoz Vásquez, G. Bélanger, J. Billard, and F. Mayet. Probing neutralino dark matter in the MSSM and the NMSSM with directional detection, in preparation.

[57] G. Belanger, F. Boudjema, A. Pukhov, and A. Semenov, MicrOMEGAs 2.0: A Program to calculate the relic density of dark matter in a generic model, Comput.Phys.Commun. 176 (2007) 367-382, hep-ph/0607059.

[58] G. Belanger, F. Boudjema, A. Pukhov, and A. Semenov, Dark matter direct detection rate in a generic model with micrOMEGAs2.1, Comput. Phys. Commun. 180 (2009) 747-767, arXiv:0803.2360.

[59] G. Belanger et. al., Indirect search for dark matter with micrOMEGAs2.4, arXiv:1004.1092.

[60] A. Djouadi, J.-L. Kneur, and G. Moultaka, SuSpect: A Fortran code for the supersymmetric and Higgs particle spectrum in the MSSM, Comput.Phys.Commun. 176 (2007) 426-455, hep-ph/0211331.

[61] A. Djouadi, M. Muhlleitner, and M. Spira, Decays of supersymmetric particles: The Program SUSY-HIT (SUspect-SdecaY-Hdecay-InTerface), Acta Phys.Polon. B38 (2007) 635-644, hep-ph/0609292.

[62] P. Bechtle, O. Brein, S. Heinemeyer, G. Weiglein, and K. E. Williams, HiggsBounds: Confronting Arbitrary Higgs Sectors with Exclusion Bounds from LEP and the Tevatron, Comput.Phys.Commun. 181 (2010) 138-167, arXiv:0811.4169.

[63] P. Bechtle, O. Brein, S. Heinemeyer, G. Weiglein, and K. E. Williams, HiggsBounds 2.0.0: Confronting Neutral and Charged Higgs Sector Predictions with Exclusion Bounds from LEP and the Tevatron, Comput.Phys.Commun. 182 (2011) 2605-2631, arXiv:1102.1898.

[64] CMS Collaboration, S. Chatrchyan et. al., Search for Neutral MSSM Higgs Bosons Decaying to Tau Pairs in pp Collisions at $\sqrt{s}=7$ TeV, Phys.Rev.Lett. 106 (2011) 231801, arXiv:1104.1619.

[65] CMS Collaboration, S. Chatrchyan et. al., Search Tau pairs, CMS PAS HIG-11-011.

[66] J. Giedt, A. W. Thomas, and R. D. Young, Dark matter, the CMSSM and lattice QCD, Phys.Rev.Lett. 103 (2009) 201802, arXiv:0907.4177.

[67] D. Albornoz Vasquez, G. Belanger, and C. Boehm, Astrophysical limits on light NMSSM neutralinos, Phys.Rev. D84 (2011) 095008, arXiv:1107.1614.

[68] J. F. Navarro, C. S. Frenk, and S. D. White, A Universal density profile from hierarchical clustering, Astrophys.J. 490 (1997) 493-508, astro-ph/9611107.

[69] D. A. Vasquez, MIMAC potential discovery and exclusion of neutralinos in the MSSM and NMSSM, arXiv:1109.3660.

[70] CMS and LHCb Collaboration, Search for the rare decay $B_{s}^{0} \rightarrow \mu^{+} \mu^{-}$at the LHC with the CMS and LHCb experiments. Combination of LHC results of the search for $B s \rightarrow \mu^{+} \mu^{-}$decays, CMS PAS BPH-11-019.

[71] A. de Roeck, "Higgs physics at the LHC and ILC." Plenary talk at 13th International Conference on Supersymmetry and Unification of Fundamental Interactions (SUSY 2005), IPPP Durham, 18-23 July 2005. 
[72] S. P. Martin and J. D. Wells, Muon anomalous magnetic dipole moment in supersymmetric theories, Phys.Rev. D64 (2001) 035003, hep-ph/0103067].

[73] M. Byrne, C. Kolda, and J. E. Lennon, Updated implications of the muon anomalous magnetic moment for supersymmetry, Phys.Rev. D67 (2003) 075004, hep-ph/0208067. 


\begin{tabular}{|c|c|c|c|c|c|c|}
\hline Parameter & A & B & $\mathrm{C}$ & $\mathrm{D}$ & $\mathrm{E}$ & $\mathrm{F}$ \\
\hline$M_{1}$ & 55 & 144.2 & 211 & 48.0 & 52.2 & 41.2 \\
\hline$M_{2}$ & 628 & 518 & 1426 & 221 & 223 & 186 \\
\hline$M_{3}$ & 1648 & 4762 & 3745 & 5596 & 4794 & 4059 \\
\hline$\mu$ & 103.2 & 114.6 & 200 & 132.2 & 124.1 & 707 \\
\hline $\tan \beta$ & 9.95 & 12.0 & 5.0 & 9.77 & 6.19 & 9.75 \\
\hline$M_{A}$ & 97.8 & 265 & 500 & 409 & 1353 & 747 \\
\hline$A_{t}$ & -1014 & -2886 & -2091 & 127 & 2112 & -390 \\
\hline$M_{\tilde{l}_{L}}$ & 114 & 157 & 239 & 235 & 197 & 77.3 \\
\hline$M_{\tilde{l}_{R}}$ & 632 & 765 & 2023 & 937 & 1657 & 1017 \\
\hline$M_{\tilde{q}_{1,2}}$ & 592.3 & 1946 & 1471 & 1789 & 1514 & 1505 \\
\hline$M_{\tilde{q}_{3}}$ & 638.8 & 1403 & 1099 & 1812 & 1959 & 1313 \\
\hline$M_{\tilde{\chi}_{1}^{0}}$ & 40.1 & 89.6 & 168 & 38.0 & 37.9 & 39.3 \\
\hline$M_{\tilde{\chi}_{1}^{+}}$ & 101.2 & 112.6 & 202 & 111.8 & 102.5 & 189.2 \\
\hline$M_{h}$ & 94.2 & 123.5 & 116.7 & 114.3 & 118.1 & 110.9 \\
\hline$R_{h g g}$ & 1.73 & 0.79 & 0.762 & 0.926 & 0.933 & 0.949 \\
\hline$R_{h b b}$ & 82.2 & 2.04 & 1.19 & 1.29 & 1.024 & 1.07 \\
\hline$R_{h W W}$ & 0.193 & 1.0 & 1.0 & 1.0 & 1.0 & 1.0 \\
\hline $\mathrm{BR}(h \rightarrow \mathrm{inv})$ & 0.04 & 0.0 & 0.0 & 0.532 & 0.734 & 0.99 \\
\hline $\mathrm{BR}(h \rightarrow \tau \bar{\tau}) / \mathrm{SM}$ & 1.03 & 1.17 & 1.02 & 0.474 & 0.261 & 0.0088 \\
\hline$R_{g g \gamma \gamma}$ & 0.008 & 0.476 & 0.704 & 0.358 & 0.257 & 0.0071 \\
\hline$R_{W W b b}$ & 0.197 & 1.17 & 1.02 & 0.473 & 0.261 & 0.0088 \\
\hline$R_{g g \tau \tau}$ & 1.78 & 0.922 & 0.775 & 0.439 & 0.243 & 0.0088 \\
\hline$R_{W W \tau \tau}$ & 0.199 & 1.17 & 1.02 & 0.474 & 0.261 & 0.0088 \\
\hline$R_{b b \tau \tau}$ & 84.8 & 2.38 & 1.21 & 0.614 & 0.267 & 0.0094 \\
\hline
\end{tabular}

Table 2: Benchmarks in the MSSM with some suppressed Higgs signal, for Benchmark F, $M_{\tilde{\nu}}=44.4 \mathrm{GeV}$. These are selected from the set of allowed points. 